\title{
Prototype Cryospheric Experimental Synthetic Aperture Radiometer (CESAR)
}

\author{
Lawrence M. Hilliard ${ }^{1}$, Norman L. Phelps ${ }^{1}$, J. Thomas Riley ${ }^{1}$, Thorsten M. Markus ${ }^{3}$ \\ Geoffrey L. Bland ${ }^{3}$, Christopher Ruf ${ }^{4}$, Roland W. Lawrence ${ }^{5}$, Steven C. Reising ${ }^{6}$, Thomas Pichel ${ }^{7}$ \\ ${ }^{1}$ Microwave Instrument Technology Branch \\ ${ }^{2}$ Instrument Systems Branch \\ ${ }^{3}$ Instrumentation Sciences Branch \\ NASA Goddard Space Flight Center \\ Greenbelt MD 20771 \\ Lawrence.M.Hilliard@nasa.gov \\ ${ }^{3}$ Thorsten.Markus@nasa.gov \\ Geoffrey.L.Bland@nasa.gov \\ ${ }^{4}$ University of Michigan, CRuf@UMich.edu \\ ${ }^{5}$ Langley Research Center, R.W.Lawrence@.nasa.gov \\ ${ }^{6}$ Colorado State University, Steven.Reising@ColoState.edu \\ ${ }^{7}$ Valparaiso University, Thomas.Pichel@valpo.edu
}

Norman.L.Phelps@nasa.gov

2John.T.Riley@nasa.gov

Abstract- Present satellite microwave radiometers typically have a coarse spatial resolution of several kilometers or more. This is only adequate only over homogenous areas. Significantly enhanced spatial resolution is critically important to reduce the uncertainty of estimated cryospheric parameters in heterogeneous and climatically-sensitive areas. Examples include: (1) dynamic sea ice areas with frequent lead and polynya developments and variable ice thicknesses, (2) mountainous areas that require improved retrieval of snow water equivalent, and (3) melting outlet glacier or ice shelf areas along the coast of Greenland and Antarctica. For these situations and many others, an Earth surface spot size of no more than $100 \mathrm{~m}$ is necessary to retrieve the information needed for significant new scientific progress, including the synthesis of field observations with satellite observations with high confidence.

At Goddard Space Flight Center, active/passive microwave remote sensing calibration and validation programs have resulted in instrumentation that uses the underside of the fuselage and wing space of Uninhabited Aerial Vehicles (UAVS) as small remote sensing platforms. This research takes advantage of prototype antennas developed for an L-Band frequency survey (nanosat), instrumentation developed for soil moisture and salinity measurements (RadSTAR), and finally a mission concept to study sea ice topography and its interaction with snow layers. The Cryospheric Experimental Synthetic Aperture Radiometer (CESAR). CESAR is a NASA proposal to fly $K$-Band and Ka-Band thinned arrays on an Uninhabited Aerial Vehicle (UAV) in order to measure at 100 meter ground resolution. Prior to the flight of CESAR, a prototype CESAR will be tested with elements that will fly at lower altitudes to begin the system level testing of the synthetic array with commercial-off-the-shelf (COTS) components. The prototype 4-element CESAR will precede the 10 element CESAR, and an L-Band version (Little CESAR) will precede that by using Ultrastable Radiometer (USR) components and techniques from the previous $S B I R$, and RadSTAR research.

The K-Band and Ka-Band COTS prototype receivers will be augmented with monolithic microwave integrated circuits (MMICs) and multi-chip module receivers to be developed at Colorado State University. These miniaturized MMIC-based receivers have been designed to be installed inside the wing of the
CESAR UAV, a specialized vehicle developed specifically for this high spatial resolution research. The antenna element positions along the wing will be monitored to allow for correction in software of deviations from a planar collecting array.

In addition to the MMIC-based receivers, CESAR will employ a correlator chip developed for the Lightweight Rainfall Radiometer (LRR). This rad-hardened low power and low mass system was developed for Synthetic Thinned Array Radiometer (STAR) systems where mass and power are minimized to result in the largest collecting aperture possible on a given platform.

\section{Introduction}

The Cryospheric Experimental Synthetic Aperture Radiometer (CESAR) will meet high-priority cryospheric science goals including measuring the high resolution details of sea ice dynamics and thickness, snow load on sea ice, monitoring ice sheet properties along complex coastal areas, and measuring fine scale snow water equivalent in mountainous areas. This radiometer is designed to achieve a spatial resolution of less than $100 \mathrm{~m}$ in order to address scientific problems that cannot be answered using currently available aircraft or satellite instrumentation. The radiometer will make use of the well-established microwave imaging frequencies of 19 and $37 \mathrm{GHz}$ at both vertical and horizontal polarization, for which several established algorithms exist to retrieve cryospheric parameters. The antenna technology will be based on proven NASA Goddard Space Flight Center (GSFC) designs (e.g., synthetic thinned array radiometer [STAR] concepts). A UAV known as the Evolved Science Platform (ESP) will be specifically, and cost-effectively, designed around the radiometer to ensure operational reliability and scientific accuracy. The proposal will include a development pathway and interim experiments to ensure a UAV and radiometer design that meets the operational requirements of 
cryospheric science; the process envisioned will include a clear path to space.

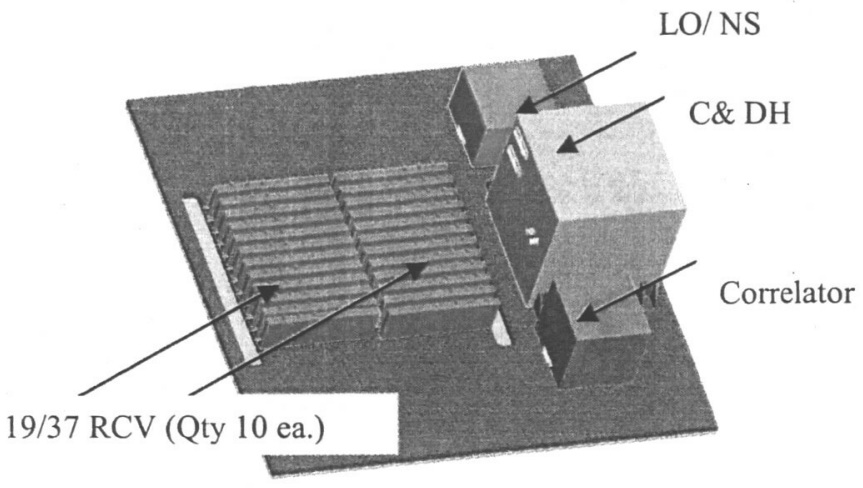

Figure 1 CESAR Components inside ESP

\section{The CESAR instrument and UAV platform}

The Prototype Cryospheric Experimental Synthetic Aperture Radiometer (CESAR) will be fabricated with discrete front end components and a patch antenna array occupying a square area approximately $11 \mathrm{~cm} \times 11 \mathrm{~cm}$. Because this version will have four receivers, it will be called the "4RCESAR." It will travel first to Colorado State University to familiarize the Monolithic Microwave Integrated Circuit (MMIC)-based radiometer developers with the system interfaces. Then the 4R-CESAR will travel to the University of Michigan to be interfaced with the correlator system under development. Finally, the 4R-CESAR will go to NASA Langley where it will be used as the core system for research and development on G-matrix tests to correct the deviations from planarity of the CESAR antenna array [2]. While CESAR is at Langley, it will be tested on a flexible panel testbed, such as is shown in Figure 2, which includes a"calibration" patch. This calibration testing will allow the CESAR team to correct for mutual coupling effects due to deformation and wing flex and to understand the dynamics of flight and their probable effects on the brightness temperature measurement. A candidate antenna element is the annular ring, which exhibits reduced surface waves, and therefore no grating lobes appear when the array is scanned far off nadir.

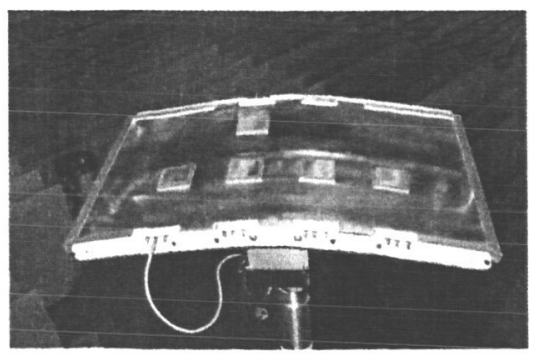

Figure 2 CESAR Wing-flex Calibration
Meanwhile, the flight system, a 10R-CESAR with MMIC Modules (shown in Figure 1) will advance at Colorado State under the direction of Dr. Steve Reising. Then the full CESAR front end will be delivered to Goddard, and integrated in the Radaire platform under development at Wallops.

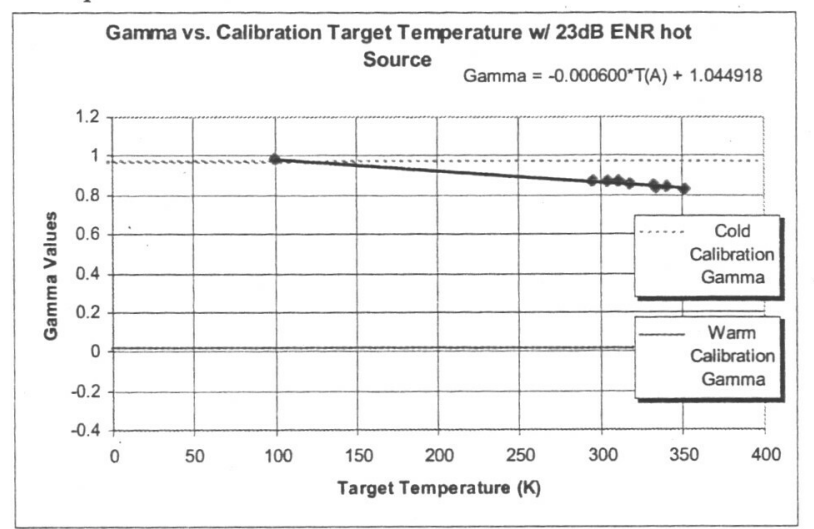

Figure 3 Linearity of NASA Goddard's DWU

Availability is a key advantage of the ESP-Lite sensing platform, and our team has L-Band components available now to pursue an L-Band version of a UAV-based radiometer. [1]

\section{The DWU and Little CESAR}

Although the proposed 4R-CESAR program has not yet been funded, a Direct-detection radiometer for the Wing of a UAV (DWU) has flown aboard the ESP airplane, and we have demonstrated its capability to match the CESAR resolution cell across a wide bandwidth and to measure a sufficient dynamic range of brightness temperatures in lab tests [3] . In figure 3 one can see that a linear relationship does indeed exist between the physical target temperature and the radiometric ratio Gamma, calculated from the ratio of radiometric counts when viewing the $150 \mathrm{~K}$ cold FET target and when viewing a $300 \mathrm{~K}$ reference, both within the DWU receiver.

Gamma is defined as:

$$
\gamma=\frac{C_{w}-C_{A}}{C_{w}-C_{c}}
$$

where $\mathrm{C}_{\mathrm{c}}$ is the number of counts when viewing the cold FET calibration source, $C_{a}$ is proportional to the Antenna Temperature, $T_{A}$, and $C_{w}$ is the number of counts when viewing the warm source.

The cold calibration gamma (dotted blue line) normalizes the counts to $\Gamma=1$ when viewing a cold FET internal to the receiver, and the warm calibration gamma normalization (solid red line, $\Gamma=0$ ) is when switching in the warm $300 \mathrm{~K}$ internal noise source. During some laboratory tests, an external "hot" noise source (+23 dB ENR) was coupled into 
the warm source at the same switch position. That combination resulted in much better linearity $(\sim 1$ order of magnitude standard deviation) over this region of equivalent target temperatures. The gamma value for the hot noise reference would be off the scale of Figure 3.

The warm noise source, by itself, is equivalent to room temperature $(\sim 300 \mathrm{~K}$ in counts). With the external hot source coupled in, as shown in Figure 5, the resultant counts yield gammas above zero for all target temperaures. However, that is attributable to system noise added by the hot source, so it can be normalized with the gamma calculation. Including a cold FET and a hot noise source in the DWU front end increases the system noise temperature somewhat, but the measurements in the lab are best calibrated with both cold and hot noise sources in the system .

Each DWU subarray is only one element wide and three elements long along the wing, and they will define a 50 meter x 100 meter ground resolution for a $50 \mathrm{~m}$ UAV flight altitude. The two DWU subarrays will view two strips of land or ocean, with a significant overlap in their ground tracks. However, the time and geolocation tagging and the video confirmation needed for its successors will be tested on the DWU.

The prototype CESAR at L-Band ("Little CESAR") will tighten the resolution cell and enable the radiometer array to scan over $+/-45$ degrees. This will be accomplished with a 6 element $\times 6$ element array integrated into the wing like CESAR. For the DWU's L-Band antenna, a $148 \mathrm{~cm}$ chord of the RadAire wing is completely utilized with 6 element spacings and 4 receivers at element positions $0,1,4$, and 6 , as shown in Figure 4 of the CESAR antenna The baseline positions of CESAR's antenna elements at the 19 and 37 $\mathrm{GHz}$ frequencies are not interleaved, so if one needs to improve the along track resolution, they can occupy adjacent locations. The MMIC/Antenna Modules (MAMs) under development at Colorado State will make the CESAR elements even smaller and make the antenna array interleaving more likely to be achieved.

\section{Other aspects of the CESAR Research Program}

Experiments at Wallops Island, Virginia are planned during May 2005 to test and calibrate the DWU over a large dynamic range of scene brightness temperatures. The first DWU flight after integration on the ESP is planned for Fall 2005 over the Delmarva peninsula. Several lightweight, radtolerant chip designs have been designed specifically for the STAR system configuration. CESAR will employ ever more antennas, resulting in better image quality, assuming 10 elements at each of $\mathrm{Ku}$ - and $\mathrm{Ka}-\mathrm{Band}$ in minimum redundancy linear array configurations [4].

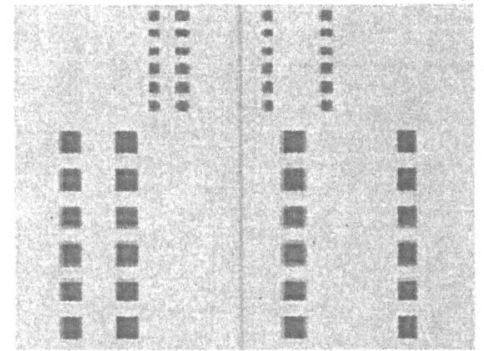

Figure 4 Prototype 4R-CESAR antennas

The results assume that the ESP is flying at an altitude of 1 $\mathrm{km}$ at a ground speed of $87 \mathrm{~km} / \mathrm{hr}(60 \mathrm{mph})$, the integration time available per linear polarization sample $(50 \%$ duty cycle) is $550 \mathrm{~ms}$ (corresponding to forward motion by onehalf of the along track antenna footprint), the pre-correlation bandwidth is $112 \mathrm{MHz}$, and the receiver noise figure is 5.0 $\mathrm{dB}$ at $\mathrm{Ku}-\mathrm{B}$ and and $5.5 \mathrm{~dB}$ at Ka-Band. Note that a constant electrical size of each aperture is maintained at both frequencies in order to match the spatial resolution between channels.

\section{Development of UAV}

A UAV that is specifically designed for radiometer instrumentation experiments will be produced using innovative packaging combined with mature construction and systems technologies. Preliminary characteristics are based on an instrument payload of $45.5 \mathrm{~kg}$ (100 lbs.), including antenna arrays, supporting electronics, and data acquisition systems. The vehicle will be configured to incorporate a rigid center section for mounting the antennas for science missions, with additional antennas and instrumentation available for engineering tests of the extended array in the outboard panels, where wing flexing effects may be more pronounced.

\section{Spaceflight Applications of CESAR and RadSTAR}

The Cold Land Processes Working Group (CLPWG) has a need for a method to close the gaps in snow and ice measurement coverages in the polar regions. The long duration ESPs, equipped with CESAR instrumentation, could fill that measurement gap.

Flying an active/passive array on a UAV is another measurement goal of GSFC's Instrumentation Sciences Branch [5]. Cross-track scanning and unfocused SAR in the along-track dimension can be co-registered with 1dimensional and 2-dimensional STAR sensors. This has been demonstrated in the L-Band Imaging Scatterometer (LIS), to be flown on the NASA P-3. Similar components for smaller research aircraft, such as UAVs, are under study. 
Complementary radar frequencies for CESAR of 13 and 17 $\mathrm{GHz}$ were recently identified by the CLPWG [6]. Adding active channels to CESAR is being considered in order to measure at higher resolution or with a wider swath.

\section{Summary}

The Cryospheric Experimental Synthetic Aperture Radiometer (CESAR) instrument incubator team has developed a method to perform high spatial resolution brightness temperature measurements using a low-flying research platform with a modest sized antenna aperture. Synthetic thinned array radiometer (STAR) technology, with an antenna integrated into the fuselage and wing of an uninhabited aerial vehicle (UAV), makes this technique transferable to higher altitudes and therefore increases the single-pass coverage area. At L-Band, the wing antenna technique is being demonstrated with the Direct-detection radiometer for the Wing of a UAV. Near term emphasis will now switch to integrating other elements of a STAR radiometer system. The next step is a prototype CESAR at L-band, "Little CESAR." This will require four Ultra-Stable Radiometers (USRs) to be flown in a thinned 6 element $\times 6$ element array. This will give NASA important flexibility to develop the required research platforms for calibration and validation of critical Earth science measurements.

\section{Acknowledgments}

Norman Phelps, Tom Riley and Thomas Pichel, formed the Goddard DWU radiometer team. Chris Ruf, Thorsten Markus, Steven Reising, Geoffrey Bland and Wes Lawrence, along with Lawrence Hilliard, comprised the CESAR proposal team. I would also like to acknowledge David Thompson who helped develop the microstrip models of the wing antenna, Ross Henry who helped with the antenna modeling, Carey Johnson, Rafael Rincon, and Mark Stephens who assisted with DWU testing, and Jim Mead and Mark Goodberlet of ProSensing who assisted the radiometer team by answering our many questions. Thanks also to Ted Miles who integrated the wing antennas and the $D W U$ in the UAV platform.

Peter Hildebrand, Terry Doiron, Cathy Long, Jeff Piepmeier, Joe Knuble, Zhaonan Zhang and Phyllis Hestnes have also supplied needed help and direction.

\section{References}

[1] Hilliard, L.M., R. Rincon, P. Hildebrand, and J. Mead, "Lightweight Linear Broadband Antennas enabling small UAV Wing Systems and Spaceflight Nanosat Concept," Proceedings of IGARSS 2004, Anchorage, Alaska, Sept. 2004.

[2] Lawrence, R.W., "Research on Reduced Surface Wave Antenna Elements, FINAL REPORT TO NASA EARTH SCIENCE

TECHNOLOGY OFFICE: Parametric Evaluation of Cold-land Processes Measurement Technologies," August 2003.

[3] Phelps, N.L., "RadSTAR Direct-Detection Wing Radiometer for an Uninhabited Aerial Vehicle (DWU)," April 29, 2005

[4] Ruf, C.S., "Numerical Anneling of Low Redundancy Linear Arrays," IEEE Trans. on Antennas and Propagation, Vol. 41, pp. 85-90, 1993.

[5] Rincon, R. P. Hildebrand, L. Hilliard, and J. Mead, "NASA's L-Band Imaging Scatterometer," Proceedings of IGARSS 2004, Anchorage, Alaska, Sept. 2004.

[6] "Meeting of the Cold Lands Processes Working Group: Radar Algorithm Meeting," February 7-1 $1^{\text {th }}, 2005$.

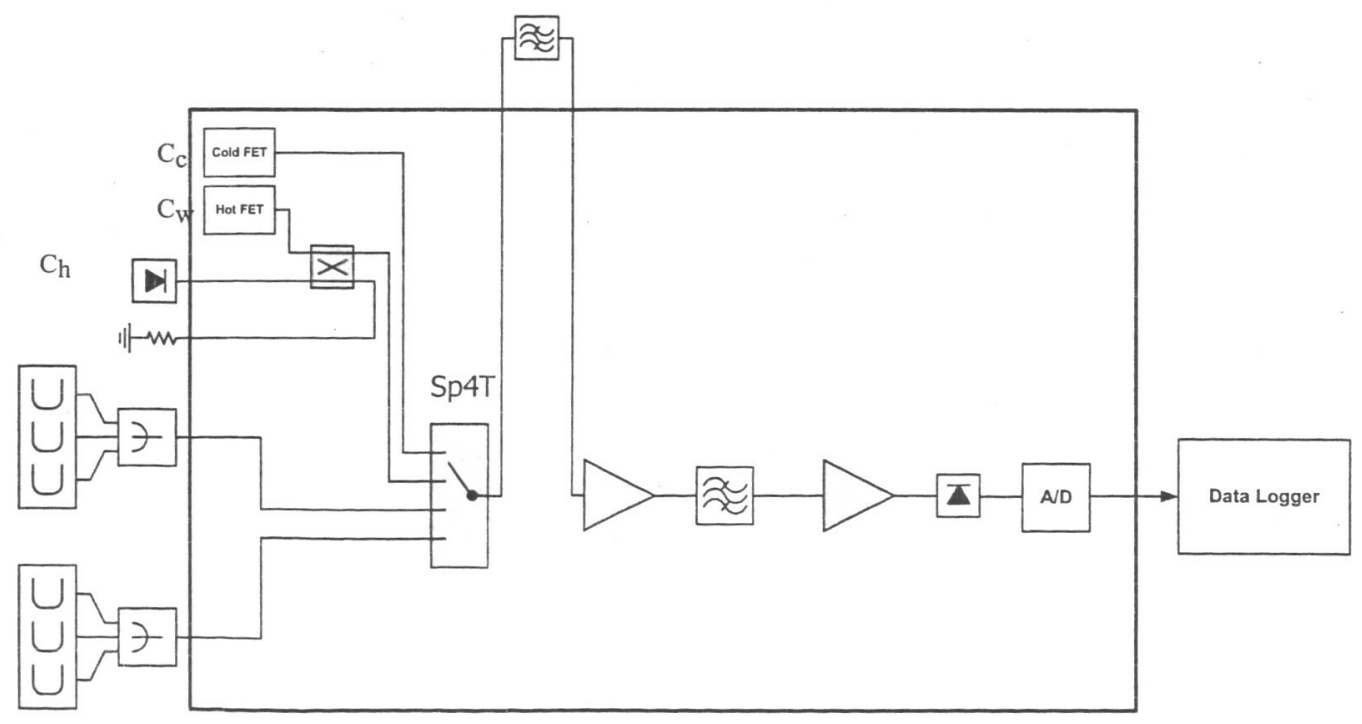

Figure 5 DWU Block Diagram 